TRANSACTIONS OF THE

AMERICAN MATHEMATICAL SOCIETY

Volume 362, Number 8, August 2010, Pages 4117-4136

S 0002-9947(10)04855-5

Article electronically published on March 17, 2010

\title{
ON NONEXISTENCE OF BARAS-GOLDSTEIN TYPE FOR HIGHER-ORDER PARABOLIC EQUATIONS WITH SINGULAR POTENTIALS
}

\author{
V. A. GALAKTIONOV AND I. V. KAMOTSKI
}

AbStract. The celebrated result by Baras and Goldstein (1984) established that the heat equation with singular inverse square potential in a smooth bounded domain $\Omega \subset \mathbb{R}^{N}, N \geq 3$, such that $0 \in \Omega$,

$$
u_{t}=\Delta u+\frac{c}{|x|^{2}} u \quad \text { in } \Omega \times(0, T),\left.\quad u\right|_{\partial \Omega}=0,
$$

in the supercritical range

$$
c>c_{\text {Hardy }}(1)=\left(\frac{N-2}{2}\right)^{2},
$$

does not have a solution for any nontrivial $L^{1}$ initial data $u_{0}(x) \geq 0$ in $\Omega$ or for a positive measure. Namely, it was proved that a regular approximation of a possible solution by a sequence $\left\{u_{n}(x, t)\right\}$ of classical solutions of uniformly parabolic equations with bounded truncated potentials given by

$$
V(x)=\frac{c}{|x|^{2}} \mapsto V_{n}(x)=\min \left\{\frac{c}{|x|^{2}}, n\right\} \quad(n \geq 1)
$$

diverges, and, as $n \rightarrow \infty$,

$$
u_{n}(x, t) \rightarrow+\infty \quad \text { in } \Omega \times(0, T) .
$$

In the present paper, we reveal the connection of this "very singular" evolution with a spectrum of some "limiting" operator. The proposed approach allows us to consider more general higher-order operators (for which Hardy's inequalities were known since Rellich, 1954) and initial data that are not necessarily positive. In particular it is established that, under some natural hypothesis, the divergence result is valid for any $2 m$ th-order parabolic equation with singular potential

$$
u_{t}=-(-\Delta)^{m} u+\frac{c}{|x|^{2 m}} u \quad \text { in } \quad \Omega \times(0, T), \quad \text { where } \quad c>c_{\mathrm{H}}(m), \quad m \geq 1,
$$

with zero Dirichlet conditions on $\partial \Omega$ and for a wide class of initial data. In particular, typically, the divergence holds for any data satisfying

$$
u_{0}(x) \text { is continuous at } x=0 \text { and } u_{0}(0)>0 .
$$

Similar nonexistence (i.e., divergence as $\varepsilon \rightarrow 0$ ) results are also derived for time-dependent potentials $\varepsilon^{-2 m} q\left(\frac{x}{\varepsilon}, \frac{t}{\varepsilon^{2 m}}\right)$ and nonlinear reaction terms $\frac{|u|^{p}}{\varepsilon^{2 m}+|x|^{2 m}}$ with $p>1$. Applications to other, linear and semilinear, Schrödinger and wave PDEs are discussed.

Received by the editors February 4, 2008.

2000 Mathematics Subject Classification. Primary 35K55, 35K40.

Key words and phrases. Parabolic equations with singular potentials, Hardy inequality, nonexistence, regular approximations, oscillatory solutions.

(C)2010 American Mathematical Society Reverts to public domain 28 years from publication 


\section{IntroduCtion: BARAS-GOLdSTEIN RESUlT AND EXTENSIONS}

This paper treats the questions of regular approximations of higher-order parabolic equations and the corresponding elliptic operators with singular unbounded potentials. There are two main generating and key ingredients of such a theory:

(i) The origin of such research can be attributed to Hardy's inequalities (19191920 ) for such symmetric operators in the $L^{2}$-space, which established the parameter supercritical range, where the operators are not semibounded and hence do not admit Friedrichs' classic self-adjoint extensions (1935). It is also crucial that, at the same time, in view of coinciding deficiency indices, there exist infinitely many other self-adjoint extensions with discrete spectra and $L^{2}$-eigenfunctions.

(ii) It was later the discovery of Baras-Goldstein (1984), that, in the corresponding nonstationary parabolic flows, regular approximations of nonnegative solutions uniformly diverge, i.e., the corresponding semigroup gives infinite values for any such nontrivial data.

It turned out later on that, in general singular linear and nonlinear PDE theory, these are important problems concerning the existence of extended semigroups of proper (blow-up) solutions that are obtained by regular approximations. For a number of parabolic, hyperbolic, and other evolution equations of mathematical physics, such semigroups can be essentially discontinuous in any suitable or admissible metric. Actually, this means that a proper solution blows up at some $t=T \geq 0$ and ceases to exist as a bounded solution for $t>T$, so a special framework of extended semigroup theory via regularization should be put in charge instead of the classic one. In particular, proper setting of basic problems such as the standard Cauchy or initial boundary value ones represents a difficult subject. Concerning related elliptic operators with singular potentials, it is then key to understand which of their spectral properties admit a proper approximation by a family of "regularized" operators with truncated singularities. It turns out that, even for classic Laplacian-type operators with inverse square potentials in the supercritical Hardy range, standard self-adjoint extensions have nothing to do with the actual operator that occurs in the regularization limit. It is revealed that this actual "limiting" operator has positive eigenvalues, and we demonstrate that they are responsible for the effective "nonexistence" of the limit of a solution. This point of view allows us not to rely on the Maximum Principle (which was a standard tool in previous work related to Baras-Goldstein type problems) and to consider much more general operators for which the Maximum Principle is not available. In particular, we demonstrate it by considering higher-order elliptic operators. Such $2 m$ th-order operators are common in PDE theory and mathematical physics, and the first derivation of Hardy's inequality for $m=2$ goes back to Rellich (1954). In what follows, we explain these aspects in greater detail, treating other PDEs as well.

Thus, one of the first key results of modern theory of singular elliptic operators and related extended discontinuous semigroups of blow-up solutions was obtained by Baras and Goldstein [1].

1.1. Baras-Goldstein (1984): Nonexistence for singular potential in linear heat equations. Let $\Omega \subset \mathbb{R}^{N}$ be a smooth bounded domain where $N \geq 3$ and $0 \in \Omega$. In Baras-Goldstein [1, the authors considered the heat equation with the inverse square potential and the zero Dirichlet boundary condition

$$
u_{t}=\Delta u+\frac{c}{|x|^{2}} u \quad \text { in } \quad \Omega \times(0, T), \quad u(x, t)=0 \text { on } \partial \Omega \times(0, T),
$$


and nonnegative initial data

$$
u(x, 0)=u_{0}(x) \geq 0 \quad \text { in } \Omega,
$$

where $u_{0} \in L^{1}(\Omega)$ or is a positive measure.

Their key nonexistence result is as follows: in the supercritical Hardy range

$$
c>c_{\mathrm{H}}=\left(\frac{N-2}{2}\right)^{2}
$$

the problem (1.1), (1.2) does not have a solution 1 Namely, this means that the regular approximation of a possible solution by the sequence $\left\{u_{n}(x, t)\right\}$ of bounded classical solutions satisfying (1.1) with the truncated bounded potentials obtained on replacement

$$
V(x)=\frac{c}{|x|^{2}} \mapsto V_{n}(x)=\min \left\{\frac{c}{|x|^{2}}, n\right\}, \quad \text { with } \quad n>0,
$$

diverges. More precisely, as $n \rightarrow \infty$,

$$
u_{n}(x, t) \rightarrow+\infty \text { in } \Omega \times(0, T) .
$$

On the contrary, for $c \leq c_{\mathrm{H}}$ in (1.1), this sequence has a finite limit that corresponds to the existence of a unique solution of the Cauchy problem (1.1), (1.2).

The results and ideas of the pioneering paper [1] generated a new direction of nonexistence/existence theory for linear and nonlinear PDEs. We refer to [17, 18, 21] for the study of linear parabolic equations and to [16, 22] which are devoted to quasilinear reaction-diffusion PDEs. These questions are reflected in the books 25 and [7, Ch. 11].

1.2. Main extensions to higher-order PDEs without positivity assumptions on data. As our main extended model, we consider the $2 m$ th-order parabolic equation with a singular potential,

(1.6) $u_{t}=\mathbf{B}_{0} u \equiv-(-\Delta)^{m} u+\frac{c}{|x|^{2 m}} u \quad$ in $\Omega \times \mathbb{R}_{+}$, where $m \geq 1, \quad N>2 m$.

For definiteness, we take zero Dirichlet conditions on the smooth boundary

$$
u=\frac{\partial u}{\partial \nu}=\ldots=\frac{\partial^{m-1} u}{\partial \nu^{m-1}}=0 \quad \text { on } \quad \partial \Omega \times \mathbb{R}_{+},
$$

where $\nu$ is the unit outward normal to $\partial \Omega$ and the initial data

$$
u(x, 0)=u_{0}(x) \in L^{2}(\Omega) .
$$

Actually, as one can expect, the nonexistence result is purely associated with the strong singularity at $x=0$ and does not essentially depend on boundary conditions (though their self-adjoint nature is a convenient assumption for calculus applied).

As in Baras-Goldstein [1, our crucial assumption is that the potential in (1.6) belongs to the supercritical range, i.e., the constant $c$ is large enough:

$$
c>c_{\mathrm{H}}(m) \text {. }
$$

Here $c_{\mathrm{H}}(m)$ for dimensions $N>2 m$ is the classic Hardy's best constant of multiplicative inequalities involving the potential in (1.6). This goes back to Hardy (1919) for $m=1$ and Rellich (1954) for $m=2$; see [13] and 29] for further references and the full history. The Hardy constant is given by

$$
c_{\mathrm{H}}(m)= \begin{cases}B_{2} B_{4} \ldots B_{m} & \text { for } m \text { even, } \\ B_{3} B_{5} \ldots B_{m} c_{\mathrm{H}}(1) & \text { for } m \text { odd }\end{cases}
$$

\footnotetext{
${ }^{1}$ The question on nonexistence was posed to the authors by H. Brezis and J.-L. Lions, 1 , p. 122].
} 
Here $B_{k}=\left[\frac{(N-2 k)(N+2 k-4)}{4}\right]^{2}$ for $k=1,2, \ldots, m$, and $c_{\mathrm{H}}(1)$ is as in (1.3); see a simple derivation and a list of references in [8, and also [5, 26] with a number of applications for $m=1$.

In a manner similar to [1] and as usual in extended semigroup theory [11, 7], we construct a proper solution of the problem (1.6)-(1.9) using regular approximations. For convenience, instead of (1.4) (clearly, this does not affect the final results), we perform an analytic approximation of the potential by replacing

$$
V(x)=\frac{c}{|x|^{2 m}} \mapsto V_{\varepsilon}(x)=\frac{c}{\varepsilon^{2 m}+|x|^{2 m}} \quad \text { with } \quad \varepsilon>0 .
$$

By $\left\{u_{\varepsilon}(x, t)\right\}$ we denote the sequence of classical bounded (for $t>0$ ) solutions of this regularized initial-value problem for the parabolic PDE with the potentials (1.11),

$$
u_{\varepsilon}: \quad u_{t}=\mathbf{B}_{\varepsilon} u \equiv-(-\Delta)^{m} u+\frac{c}{\varepsilon^{2 m}+|x|^{2 m}} u \quad \text { in } \quad \Omega \times \mathbb{R}_{+} \quad(m \geq 1, \quad N>2 m) .
$$

We then take the same Dirichlet boundary conditions (1.7) and the same initial data (1.8).

Passing to the limit as $\varepsilon \rightarrow 0$ is performed in Section 3 , which is based on a more general approach in Section 2 to $2 m$ th-order parabolic equations with arbitrary potentials. In particular, we show that the phenomenon

$$
\left\{u_{\varepsilon}(x, t)\right\} \text { diverges in } L^{2}(\Omega) \text { as } \varepsilon \rightarrow 0
$$

is a generic (robust) property of such approximations of singular parabolic problems.

As a simpler counterpart, it follows that for $c \leq c_{\mathrm{H}}$ the limit of $\left\{u_{\varepsilon}\right\}$ always exists,

$$
u_{\varepsilon}(x, t) \rightarrow \bar{u}(x, t) \quad \text { as } \quad \varepsilon \rightarrow 0
$$

for any data $u_{0} \in L^{2}(\Omega)$, where $\bar{u}(\cdot, t) \in L^{2}(\Omega)$ for all $t>0$. This means the existence of a (unique) solution.

The nonexistence result (1.13) actually means that, in the supercritical range $c>c_{\mathrm{H}}$,

$$
|\bar{u}(x, t)|=\infty
$$

can be treated as a "proper solution" of the original problem (1.6)-(1.8) for any $L^{2}$ data satisfying some extra conditions, e.g., the regularity and positivity at the origin (or, more generally, nonorthogonality to a positive lineal; see below). Then, as we mentioned at the beginning of Introduction, (1.15) means that the extended semigroup of such solutions obtained by regular approximations is discontinuous at $t=0$ for sufficiently arbitrary initial data $u_{0}$. Note that the behaviour of the "approximating" family $\left\{u_{\varepsilon}(x, t)\right\}$ as $\varepsilon \rightarrow 0$ to get (1.15) can be extremely oscillatory. For instance, for data $u_{0}(x)$ that are oscillatory at $x=0$, we present in Section 4 an example of radial solutions with nonuniform oscillatory blow-up, where $u_{\varepsilon}(x, t)$ has both limits $\pm \infty$ along some subsequences, and the same holds even at the origin $x=0$.

In the present paper, we study the case where the singularity of the potential is concentrated at the single internal point $x=0 \in \Omega$, and this indeed affects our final nonexistence conclusions. We do not consider the case of singularities on the boundary, which can lead to other regularization spectral properties and hence different and more difficult nonexistence criteria. In the elliptic case, there exist 
related extended Hardy's inequalities for smooth domains $\Omega$ corresponding to potentials with inverse square singularity on the boundary $\partial \Omega$, which were introduced in [4]. See also [19], where further extensions via perturbations of such potentials were presented, and also [14, 15] for delicate nonlinear counterparts.

1.3. Layout of the paper. Sections 26 are occupied with various aspects of nonexistence analysis of parabolic models such as (1.6) and their time-dependent and nonlinear extensions. To show further application, in Section 7 we discuss some rather simple corollaries of our analysis being applied to Schrödinger and hyperbolic PDEs such as

$$
\mathrm{i} u_{t}=-(-\Delta)^{m} u+\frac{c}{|x|^{2 m}} u \quad \text { and } \quad u_{t t}=-(-\Delta)^{m} u+\frac{c}{|x|^{2 m}} u,
$$

together with their semilinear counterparts.

\section{General nonexistence theorem}

2.1. Divergence theorem for a general potential. In this section, we treat the nonexistence in a more general setting. We consider the Cauchy-Dirichlet problem for the poly-harmonic equation with a general regularized potential,

$$
u_{\varepsilon}: \quad u_{t}=\mathbf{B}_{\varepsilon} u \equiv-(-\Delta)^{m} u+\frac{1}{\varepsilon^{2 m}} q\left(\frac{x}{\varepsilon}\right) u \quad \text { in } \quad \Omega \times \mathbb{R}_{+} \quad(m \geq 1) .
$$

Here the potential $q_{\varepsilon}(x) \equiv \frac{1}{\varepsilon^{2 m}} q\left(\frac{x}{\varepsilon}\right)$ depends on the parameter $\varepsilon>0$, for instance, in a manner similar to that in (1.12), where $q(y)$ is now an arbitrary smooth function in $\mathbb{R}^{N}$ decaying at infinity. Therefore, (2.1) plays the role of a regular approximation of the parabolic equation with the singular potential $V(x)(V(0)=\infty$ and $|V(x)|<$ $\infty$ for $x \neq 0$ ) such that, uniformly on compact subsets in $\mathbb{R}^{N} \backslash\{0\}$ and sufficiently fast,

$$
q_{\varepsilon}(x)=\frac{1}{\varepsilon^{2 m}} q\left(\frac{x}{\varepsilon}\right) \rightarrow V(x) \quad \text { as } \quad \varepsilon \rightarrow 0^{+} \quad\left(\text { e.g., } V(x)=\frac{c}{|x|^{2 m}} \text { as in (1.6) }\right) .
$$

We assume for $u_{\varepsilon}(x, t)$ zero Dirichlet conditions on the smooth boundary,

$$
u_{\varepsilon}=\frac{\partial u_{\varepsilon}}{\partial \nu}=\ldots=\frac{\partial^{m-1} u_{\varepsilon}}{\partial \nu^{m-1}}=0 \quad \text { on } \quad \partial \Omega \times \mathbb{R}_{+},
$$

where $\nu$ is the unit outward normal to $\partial \Omega$, and we prescribe initial data

$$
u_{\varepsilon}(x, 0)=u_{0 \varepsilon}(x) \in L^{2}(\Omega)
$$

that, in general, also depend on $\varepsilon$.

In what follows, $C$ and $c_{\varepsilon}$ denote various positive constants whose exact values are of no importance. Here $C$ is independent of $\varepsilon$, while constants $c_{\varepsilon}$ have at most rational dependence on $\varepsilon$, i.e.,

$$
\varepsilon^{P}<c_{\varepsilon}<\varepsilon^{-P}
$$

for some positive $P$ which can be arbitrarily large. In this calculus, we easily write $c_{\varepsilon} c_{\varepsilon} \geq c_{\varepsilon}$. Such constants are not of importance and are negligible when dealing with exponential factors such as $\mathrm{e}^{1 / \varepsilon}, \mathrm{e}^{1 / \varepsilon^{2}}$, or $\mathrm{e}^{1 / \varepsilon^{2 m}}$. The latter are to be treated more carefully.

First of all, using the above calculus, since the equation is linear, we may always assume that

$$
c_{\varepsilon} \leq\left\|u_{0 \varepsilon}\right\|_{L^{2}} \leq c_{\varepsilon} \quad \text { for } \varepsilon>0 \text { small; }
$$

i.e., $u_{0 \varepsilon}$ does not get large as $\varepsilon \rightarrow 0$, which is a natural and not restrictive assumption. 
Our main hypothesis on the potential is as follows:

Hypothesis (P). The limiting operator

$$
\mathbf{B} \equiv-\left(-\Delta_{y}\right)^{m}+q(y) I \quad \text { in } \mathbb{R}^{N}
$$

has $M \geq 1$ positive eigenvalues with exponentially decaying eigenfunctions, and, for some its eigenfunction $U_{n}(y)$,

$$
\left|\left\langle u_{0 \varepsilon}(x), U_{n}\left(\frac{x}{\varepsilon}\right)\right\rangle\right| \geq c_{\varepsilon} \quad \text { for all small } \varepsilon>0 .
$$

In the following particular cases, (2.7) is replaced by:

(i) if $u_{0 \varepsilon}(\varepsilon x) \rightarrow \delta_{0}>0$ as $\varepsilon \rightarrow 0$ (e.g., $u_{0}$ is independent of $\varepsilon, u_{0}(x)$ is continuous at $x=0$ and $\left.u_{0}(0)=\delta_{0}>0\right)$, there exists $U_{n}(y)$ that has nonzero mean in $\mathbb{R}^{N}$, and

(ii) if $u_{0 \varepsilon}(x)$ is supported in some ball $B_{\bar{c}_{\varepsilon}}(0)$, with $\frac{\bar{c}_{\varepsilon}}{\varepsilon} \rightarrow 0$ and $\int u_{0 \varepsilon}(x) \geq c_{\varepsilon}$, there exists an eigenfunction $U_{n}(y)$ of (2.6) that does not vanish at the origin $y=0$.

Our main nonexistence result is a corollary of the following estimate:

Theorem 2.1. Let $m \geq 1$, (1.9), and Hypothesis (P) hold. Then the sequence $\left\{u_{\varepsilon}(x, t)\right\}$ of classical solutions of the approximating problem (2.1)-(2.4) diverges in $L^{2}(\Omega)$ as $\varepsilon \rightarrow 0$ :

$$
\left\|u_{\varepsilon}(x, t)\right\|_{L^{2}(\Omega)} \geq \mathrm{e}^{\frac{C}{\varepsilon^{2 m}} t} \rightarrow \infty \text { for any fixed } t>0 .
$$

2.2. Proof of Theorem 2.1. Fix a sufficiently small $\varepsilon>0$ and consider the corresponding eigenvalue problem

$$
\mathbf{B}_{\varepsilon} \psi=\lambda \psi, \quad \psi \in H^{2 m}(\Omega) \cap H_{0}^{m}(\Omega) .
$$

By $\sigma\left(\mathbf{B}_{\varepsilon}\right)=\left\{\lambda_{j}^{\varepsilon}, j \geq 0\right\}$ and $\left\{\psi_{j}^{\varepsilon}, j \geq 0\right\}$ we denote the corresponding spectrum and the orthonormal, complete, and closed in $L^{2}(\Omega)$ eigenfunctions subset, 2]. Clearly,

$$
\begin{gathered}
u_{\varepsilon}(x, t)=\sum_{(j \geq 0)} c_{j}^{\varepsilon} \psi_{j}^{\varepsilon}(x) \mathrm{e}^{\lambda_{j}^{\varepsilon} t}, \text { with } c_{j}^{\varepsilon}=\left\langle u_{0 \varepsilon}, \psi_{j}^{\varepsilon}\right\rangle, \\
\text { so that }\left\|u_{\varepsilon}(\cdot, t)\right\|_{L^{2}(\Omega)}^{2}=\sum_{(j \geq 0)}\left|c_{j}^{\varepsilon}\right|^{2} \mathrm{e}^{2 \lambda_{j}^{\varepsilon} t} .
\end{gathered}
$$

We will need the following statement:

Lemma 2.1. Let $\Lambda_{j}, U_{j}$ for $j=1,2, \ldots, M$, be positive eigenvalues and corresponding normalized eigenfunctions of (2.6). Then, for any small enough $\varepsilon>0$, intervals

$$
\left[\frac{\Lambda_{j}}{\varepsilon^{2 m}}-\mathrm{e}^{-\frac{C}{\varepsilon}}, \frac{\Lambda_{j}}{\varepsilon^{2 m}}+\mathrm{e}^{-\frac{C}{\varepsilon}}\right]
$$

contain eigenvalues of the problem (2.9). Moreover, the function $U_{j}^{\varepsilon}=U_{j}\left(\frac{x}{\varepsilon}\right)$ is an "approximate" eigenfunction of (2.9) in the following sense:

$$
\left\|U_{j}^{\varepsilon}-\sum_{(1)} \alpha_{j} \psi_{j}^{\varepsilon}\right\|_{H^{m}(\Omega)} \leq \mathrm{e}^{-\frac{C}{\varepsilon}},
$$

where $\sum_{(1)}$ stands for the summation with respect to $j$ such that $\lambda_{j}^{\varepsilon}$ belongs to the above interval, and $\sum_{(1)} \alpha_{j}^{2}=c_{\varepsilon}$, with $\alpha_{j}$ being constants (depending on $\varepsilon$ ).

Let us return to the proof of the theorem. Due to the assumptions of the theorem, we fix the necessary eigenfunction $U_{n}$ of the operator (2.6) that provides us with the estimate

$$
\left|\left\langle u_{0 \varepsilon}, U_{n}\right\rangle\right| \geq c_{\varepsilon}
$$


On the other hand, due to the above lemma, we see that there is at least one eigenfunction $\psi_{l}^{\varepsilon}$ of (2.18) (see below) with the corresponding eigenvalue $\lambda_{l}^{\varepsilon}$ in the interval

$$
\lambda_{l}^{\varepsilon} \in\left[\frac{\Lambda_{j}}{\varepsilon^{2 m}}-C \mathrm{e}^{-\frac{C}{\varepsilon}}, \frac{\Lambda_{j}}{\varepsilon^{2 m}}+C \mathrm{e}^{-\frac{C}{\varepsilon}}\right] .
$$

In view of (2.12) we have

$$
\left|\left\langle u_{0 \varepsilon}, U_{n}\right\rangle\right|-\mathrm{e}^{-\frac{C}{\varepsilon}}\left\|u_{0 \varepsilon}\right\| \leq\left|\sum_{(1)} \alpha_{l}\left\langle u_{0 \varepsilon}, \psi_{l}^{\varepsilon}\right\rangle\right| \leq\left(\sum_{(1)} \alpha_{l}^{2}\right)^{\frac{1}{2}}\left(\sum_{(1)}\left|\left\langle u_{0 \varepsilon}, \psi_{l}^{\varepsilon}\right\rangle\right|^{2}\right)^{\frac{1}{2}},
$$

and consequently, (2.5) and (2.13) immediately imply that

$$
\sum_{(1)}\left|c_{l}^{\varepsilon}\right|^{2} \geq c_{\varepsilon}
$$

Then obviously we have

$$
\left\|u_{\varepsilon}(x, t)\right\|_{L_{2}(\Omega)}^{2}=\sum_{(j \geq 0)}\left|c_{l}^{\varepsilon}\right|^{2} \mathrm{e}^{2 \lambda_{l}^{\varepsilon} t} \geq \sum_{(1)}\left|c_{l}^{\varepsilon}\right|^{2} \mathrm{e}^{2 \lambda_{l}^{\varepsilon} t} \geq c_{\varepsilon} \mathrm{e}^{\frac{2 \Lambda_{j} t}{\varepsilon^{2} m}} \sum_{(1)}\left|c_{l}^{\varepsilon}\right|^{2} \geq c_{\varepsilon} \mathrm{e}^{\frac{2 C t}{\varepsilon^{2 m}}}
$$

This concludes the proof.

2.3. On generic asymptotic behaviour as $\varepsilon \rightarrow 0$. The following sharp "pointwise" estimate of the solution sequence $\left\{u_{\varepsilon}\right\}$ holds: if (2.7) is valid for $j=0$, then, for arbitrarily small fixed $t>0$,

$$
\mathrm{e}^{-\lambda_{0}^{\varepsilon} t} u_{\varepsilon}(x, t)-c_{0}^{\varepsilon} \psi_{0}^{\varepsilon}(x) \rightarrow 0 \quad \text { as } \varepsilon \rightarrow 0 \quad \text { in } L^{2}(\Omega) \quad\left(\psi_{0}^{\varepsilon}(x) \sim \varepsilon^{-\frac{N}{2}} U_{0}\left(\frac{x}{\varepsilon}\right)\right) .
$$

2.4. A weaker blow-up hypothesis. The blow-up result (2.8) remains valid under the weaker condition on initial data: (2.5) holds and

$$
\left|\left\langle u_{0 \varepsilon}(x), U_{j}\left(\frac{x}{\varepsilon}\right)\right\rangle\right| \geq \mathrm{e}^{-\frac{c_{*}}{\varepsilon}} \text { for some positive constant } c_{*}<c_{j},
$$

where the constant $c_{j}$ gives the rate of exponential decay of the eigenfunction $U_{n}$, i.e.,

$$
\left\|U_{j}(y) \mathrm{e}^{c_{j}|y|}\right\|_{H^{m}\left(\mathbb{R}^{N}\right)}<C, \quad \text { with } \quad c_{j}>0 .
$$

Assumption (2.16) is obviously satisfied in the pioneering original paper [1 for $j=0$; see Proposition 3.1 (iii).

2.5. Proof of Lemma 2.1. Consider eigenvalues and eigenfunctions of operator (2.6) $\Lambda_{j}, U_{j}, j=1,2, \ldots, M$. These eigenfunctions decay exponentially at infinity; see (2.17). Then functions $U_{j}\left(\frac{x}{\varepsilon}\right)$ satisfy the equation

$$
\mathbf{B}_{\varepsilon} U_{j}\left(\frac{x}{\varepsilon}\right)=\frac{1}{\varepsilon^{2 m}} \Lambda_{j} U_{j}\left(\frac{x}{\varepsilon}\right) \text { in } \Omega .
$$

These do not satisfy boundary conditions on $\partial \Omega$. To fix this, consider the functions

$$
V_{j}(x)=U_{j}\left(\frac{x}{\varepsilon}\right) \chi(x),
$$

where $\chi$ is a smooth function which is equal to 1 in a neighborhood of the origin and is equal to zero in some neighborhood of the boundary $\partial \Omega$. Then

$$
\begin{gathered}
\mathbf{B}_{\varepsilon} V_{j}=\frac{1}{\varepsilon^{2 m}} \Lambda_{j} V_{j}+\tilde{V}_{j} \quad \text { in } \Omega, \quad V_{j} \in H^{2 m}(\Omega) \cap H_{0}^{m}(\Omega), \quad \text { and } \\
\left\|\tilde{V}_{j}\right\|_{H^{m}(\Omega)} \leq \mathrm{e}^{-\frac{C}{\varepsilon}} .
\end{gathered}
$$

Then the application of the "Lemma on approximate eigenfunction" (see 27]) and the observation that the difference between $U_{j}$ and $V_{j}$ can be estimated via the right-hand side of (2.20) deliver the result of the lemma. 
Lemma 2.1 can be strengthened (though, in this form, we are not going to use it):

Proposition 2.1. Let $\Lambda_{j}$ and $U_{j}$ be eigenvalues and eigenfunctions of the problem (2.6). Then eigenvalues and eigenfunctions of the eigenvalue problem (2.9) satisfy: for any fixed $1 \leq j \leq M$, as $\varepsilon \rightarrow 0$,

$$
\left|\lambda_{j}^{\varepsilon}-\frac{\Lambda_{j}}{\varepsilon^{2 m}}\right| \leq C \mathrm{e}^{-c\left|\Lambda_{j}\right|^{1 / 2 m} \frac{1}{\varepsilon}}, \quad\left\|\psi_{j}^{\varepsilon}-\sum_{\left(\kappa_{j}\right)} \alpha_{k}^{\varepsilon} U_{k}\left(\frac{x}{\varepsilon}\right)\right\|_{L^{2}(\Omega)} \leq C \mathrm{e}^{-c\left|\Lambda_{j}\right|^{1 / 2 m} \frac{1}{\varepsilon}},
$$

where the summation in $\sum_{\left(\kappa_{j}\right)}$ takes place over $k$ such that $\Lambda_{k}=\Lambda_{j}$, and $\sum_{\left(\kappa_{j}\right)}\left(\alpha_{k}^{\varepsilon}\right)^{2}$ $=c_{\varepsilon}$.

\section{Problem for (1.12): Scaling, limit problem, and limit operator}

We now explain in greater detail the application of the above general results to our main model equation (1.6) with the approximation (1.12).

3.1. Scaling. Thus, we perform in (1.12) the natural scaling of the independent variables

$$
x=\varepsilon y, \quad t=\varepsilon^{2 m} \tau .
$$

This reduces (1.12) to the equation with the regular potential,

$$
u_{\tau}=\mathbf{B}_{1} u \equiv-(-\Delta)^{m} u+\frac{c}{1+|y|^{2 m}} u \quad \text { in } \quad \Omega_{\varepsilon} \times \mathbb{R}_{+},
$$

which is posed in the domain

$$
\Omega_{\varepsilon}=\left\{y \in \mathbb{R}^{N}: \quad \varepsilon y \in \Omega\right\} \quad\left(\left.u\right|_{\partial \Omega_{\varepsilon}}=0\right) .
$$

The initial data are now

$$
u_{0 \varepsilon}(x) \mapsto u_{0 \varepsilon}(\varepsilon y) \text { in } \Omega_{\varepsilon} .
$$

3.2. Limit problem. The above parabolic problems (3.2)-(3.4) are posed in a family of expanding domains $\left\{\Omega_{\varepsilon}\right\}$ that embrace the whole $\mathbb{R}^{N}$ as $\varepsilon \rightarrow 0$. Therefore, it is natural to consider the limit problem, which is the Cauchy problem for

$$
v_{\tau}=\mathbf{B}_{1} v \equiv-(-\Delta)^{m} v+\frac{c}{1+|y|^{2 m}} v \quad \text { in } \quad \mathbb{R}^{N} \times \mathbb{R}_{+} .
$$

3.3. Spectral properties of the limit operator. The limit parabolic problem for (3.5) introduces the limit operator

$$
\mathbf{B}_{1}=-(-\Delta)^{m}+\frac{c}{1+|y|^{2 m}} I \quad \text { in } \quad L^{2}\left(\mathbb{R}^{N}\right) \text {, where } \quad c>c_{\mathrm{H}}(m) .
$$

Obviously, (3.6) is semibounded,

$$
\mathbf{B}_{1} \leq c I \quad \text { in } \quad C_{0}^{\infty}\left(\mathbb{R}^{N}\right),
$$

so it admits Friedrichs' self-adjoint extension (denoted again by $\mathbf{B}_{1}$ ) [2] with the domain $H^{2 m}\left(\mathbb{R}^{N}\right)$ in view of the embedding [23, p. 54]

$$
\int_{\mathbb{R}^{N}} \frac{w^{2}}{1+|y|^{2 m}} \leq C \int_{\mathbb{R}^{N}}\left|D^{m} w\right|^{2} \quad \text { in } \quad H^{m}\left(\mathbb{R}^{N}\right) .
$$


For convenience, we present a detailed description of the necessary spectral properties:

Proposition 3.1. (i) The spectrum of the operator (3.6) with domain $H^{2 m}\left(\mathbb{R}^{N}\right)$ comprises the continuous and the discrete ones,

$$
\begin{gathered}
\sigma\left(\mathbf{B}_{1}\right)=\sigma_{c}\left(\mathbf{B}_{1}\right) \cup \sigma_{p}\left(\mathbf{B}_{1}\right)=\{\lambda \leq 0\} \cup\left\{\lambda=\Lambda_{j}>0, j=0,1,2, \ldots\right\}, \quad \text { with } \\
c>\Lambda_{0} \geq \Lambda_{1} \geq \Lambda_{2} \geq \ldots \geq \Lambda_{k} \geq \Lambda_{k+1} \geq \ldots 0,
\end{gathered}
$$

where each eigenvalue is repeated as many times as its finite multiplicity.

(ii) Each eigenfunction $U_{j}(y)$ is exponentially decaying at infinity,

$$
\left|U_{j}(y)\right| \leq A_{j} \mathrm{e}^{-\alpha_{m} \Lambda_{j}^{1 / 2 m}|y|} \quad \text { as } y \rightarrow \infty,
$$

where $A_{j}$ and $\alpha_{m}$ are positive constants.

(iii) The first eigenfunction $U_{0}(y)$ is radially symmetric. For $m=1$, it is positive,

$$
U_{0}(y)>0 \quad \text { in } \quad \mathbb{R}^{N} \quad(m=1) .
$$

For any $m \geq 2, U_{0}(y)$ has infinitely many sign changes.

Proof. Part (i). Continuous Spectrum. Fix some $\Lambda=-\mu^{2 m}<0$ and consider

$$
\mathbf{B}_{1} U=\Lambda U=-\mu^{2 m} U \text { in } \mathbb{R}^{N} .
$$

Using the Fourier Transform, it is not difficult to see that (3.13) has a radial solution with the following asymptotic behaviour: as $|y| \rightarrow \infty$,

$$
U_{\Lambda}(y) \sim|y|^{-\frac{N-2 m}{2}} \cos \left(\mu|y|+a_{0}\right) \quad\left(a_{0} \in \mathbb{R}\right) .
$$

For $m=1, U_{\Lambda}(y)$ is given by Bessel's function $J_{\nu}(\mu|y|)$, with $\nu=\frac{N-2}{2}$, [28, $\left.\S 23\right]$. Here, $U_{\Lambda} \notin H^{2 m}\left(\mathbb{R}^{N}\right)$ (and $\notin H^{m}\left(\mathbb{R}^{N}\right), \notin L^{2}\left(\mathbb{R}^{N}\right)$ ). Hence, $\left(\mathbf{B}_{1}-\Lambda I\right)^{-1}$ is not bounded for any $\lambda<0$, from whence $0 \in \sigma_{c}\left(\mathbf{B}_{1}\right)$ by closure.

Discrete SPECTRUM. We first discuss a general representation of all the eigenfunctions, which we will use later on. To this end, we introduce the polar coordinates $x=(r, \sigma)$ in $\mathbb{R}^{N}$, so that

$$
\Delta=\Delta_{r}+\frac{1}{r^{2}} \Delta_{\sigma}, \quad \text { where } \quad \Delta_{r}=D_{r}^{2}+\frac{N-1}{r} D_{r}
$$

and $\Delta_{\sigma}$ is the Laplace-Beltrami operator on the unit sphere $S^{N-1}$ in $\mathbb{R}^{N}$, which is a regular operator with discrete spectrum in $L^{2}\left(S^{N-1}\right)$ (each eigenvalue repeated as many times as its multiplicity),

$$
\sigma\left(\Delta_{\sigma}\right)=\left\{-\mu_{k} \equiv-k(k+N-2) \leq 0, k \geq 0\right\} .
$$

$\Delta_{\sigma}$ has an orthonormal, complete, and closed subset $\left\{f_{k}(\sigma)\right\}$ of eigenfunctions being $k$ th order homogeneous harmonic polynomials restricted to $S^{N-1}$.

Consider the eigenvalue problem for the operator (3.2),

$$
\begin{gathered}
\mathbf{B}_{1} U=(-1)^{m+1}\left(\Delta_{r}+\frac{1}{r^{2}} \Delta_{\sigma}\right)^{m} U+\frac{c}{1+|y|^{2 m}} U \\
\equiv(-1)^{m+1} \sum_{l=0}^{m} C_{m}^{l} \Delta_{r}^{l}\left(\frac{1}{r^{2}} \Delta_{\sigma}\right)^{m-l} U+\frac{c}{1+r^{2 m}} U=\Lambda U,
\end{gathered}
$$

where $C_{m}^{l}$ are binomial coefficients. Performing in (3.17) the separation of variables

$$
U(r, \sigma)=\phi(r) f_{k}(\sigma)
$$

yields the purely radial eigenvalue problem for $\phi(r)$,

$$
(-1)^{m+1} \sum_{l=0}^{m} C_{m}^{l}\left(-\mu_{k}\right)^{m-l} \Delta_{r}^{l}\left(\frac{1}{r^{2(m-l)}} \phi\right)+\frac{c}{1+r^{2 m}} \phi=\Lambda \phi
$$


Notice that the first operator in (3.19) contains singular potentials up to the leading singularity in the last term

$$
\ldots-\frac{c_{2 m}}{r^{2 m}} \phi, \quad \text { with a constant } \quad c_{2 m}=\mu_{k}^{m}>0 .
$$

This and other singular terms have the right sign to guarantee that this operator is negative and coercive, and, of course, this essentially affects the final result.

The positive lineal $\mathcal{L}_{+}=\operatorname{Span}\left\{U_{0}, U_{1}, \ldots\right\}$ is infinite-dimensional. The rest of the results are standard in elliptic theory; see 2]. In particular, the existence of a countable set of eigenvalues follows from the fact that the subspace on which the quadratic form for the operator (3.6),

$$
\mathbf{Q}_{1}(v)=\left\langle v, \mathbf{B}_{1} v\right\rangle,
$$

is positive, is infinite-dimensional. Indeed, let $\chi(t) \in C_{0}^{\infty}(\mathbb{R})$ be the cut-off function,

$$
\chi(t)= \begin{cases}1, & 0<t<1 \\ 0, & t>2\end{cases}
$$

and set

$$
\chi_{a, b}(t)=\chi(t-b)[1-\chi(t-a)], 0<a<b \Longrightarrow \operatorname{supp} \chi_{a, b} \in[a, b+2] .
$$

We next chose a test sequence for Rayleigh quotient $[2$ in the following way:

$$
u_{a, b}(|x|)=|x|^{-\frac{N-2 m}{2}} \chi_{a, b}(|x|) .
$$

Then we have

$$
\begin{gathered}
\mathbf{Q}_{1}\left(u_{a, b}\right)=\left\langle u_{a, b}, \mathbf{B}_{1} u_{a, b}\right\rangle \\
=\left\langle u_{a, b},\left(\frac{c}{1+|x|^{2 m}}-\frac{c_{\mathrm{H}}(m)}{|x|^{2 m}}\right) u_{a, b}\right\rangle+\left\langle u_{a, b},\left[-(-\Delta)^{m}, \chi_{a, b}\right]|x|^{-\frac{N-2 m}{2}}\right\rangle,
\end{gathered}
$$

where $[\cdot, \cdot]$ is the commutator. Here, we have used the fact that (see Section 4)

$$
-(-\Delta)^{m}|x|^{-\frac{N-2 m}{2}}=\frac{c_{H}}{|x|^{2 m}}|x|^{-\frac{N-2 m}{2}},
$$

so that the function $\left[-(-\Delta)^{m}, \chi_{a, b}\right]$ in (3.22) has support restricted to outer and inner layers according to (3.21). Obviously, the first term on the right-hand side in (3.22) tends to $+\infty$ as $b \rightarrow+\infty$ since $c>c_{\mathrm{H}}(m)$ and

$$
\int_{|x|>a+1}|x|^{-2 m}|x|^{-2 \frac{N-2 m}{2}} \mathrm{~d} x \sim \int^{\infty} r^{N-1} r^{-N} \mathrm{~d} r=+\infty .
$$

In contrast, the second term on the right-hand side in (3.22) remains bounded, since

$$
\operatorname{supp}\left[-(-\Delta)^{m}, \chi_{a, b}\right] \subset\{a \leq|x| \leq a+1\} \cup\{b+1 \leq|x| \leq b+2\} .
$$

As a result, $\mathbf{Q}_{1}\left(u_{a, b}\right) \rightarrow+\infty$ as $b \rightarrow+\infty$, and there is a $b(a)$ such that $\mathbf{Q}_{1}\left(u_{a, b(a)}\right)>0$ and supp $u_{a, b} \in\{a \leq|x| \leq b+2\}$. Now choosing a sequence $\left\{a_{i}\right\}$ such that $a_{i}>b\left(a_{i-1}\right)+2$ for $i=1,2, \ldots$, we obtain the infinite sequence $\left\{u_{a_{i}, b\left(a_{1}\right)}\right\}$ such that $\mathbf{Q}_{1}\left(u_{a_{i}, b\left(a_{i}\right)}\right)>0$ and supp $u_{a_{i}, b\left(a_{i}\right)} \cap \operatorname{supp} u_{a_{j}, b\left(a_{j}\right)}=\emptyset$ for $i \neq j$. So there is an infinite-dimensional subspace on which the quadratic form for the operator (3.6) is positive, and consequently we have infinitely many positive eigenvalues of $\mathbf{B}_{1}$.

Part (ii). Exponential decay follows from separation of variables and ODE techniques.

Part (iii). The positivity (3.12) for $m=1$ is Jentzsch's classic theorem (1912). 
3.4. Nonexistence theorem. Thus, we consider the Cauchy-Dirichlet problem for (1.12) with general data (2.4). Without loss of generality, we state the following nonexistence (divergence) result, where, for convenience, we slightly change the argument of the proof. This underlines extra features of the above positive lineal of the limit operator (3.6),

$$
\mathcal{L}_{+}=\operatorname{Span}\left\{U_{0}, U_{1}, \ldots\right\},
$$

as a set of all finite linear combinations of the given eigenfunctions.

Theorem 3.1. Let

$$
u_{0 \varepsilon}(\varepsilon y) \rightarrow v_{0}(y) \quad \text { as } \varepsilon \rightarrow 0 \text { in } L_{\mathrm{loc}}^{2}\left(\mathbb{R}^{N}\right),
$$

and let it be, in the metric of $L^{2}\left(\mathbb{R}^{N}\right)$, that

$$
v_{0} \text { is not orthogonal to } \mathcal{L}_{+} \text {. }
$$

Then (2.8) holds.

The proof repeats the arguments of the proof of Theorem 2.1. In particular, assuming the continuity and strict positivity at the origin of the data that are independent of $\varepsilon$,

$$
u_{0 \varepsilon}(x)=u_{0}(x) \quad \text { in } \quad \Omega \quad \text { and } \quad u_{0} \in C\left(B_{1}\right), u_{0}(0)=\delta_{0}>0,
$$

we obtain from (3.24) that

$$
v_{0}(y) \equiv \delta_{0}=u_{0}(0)>0 .
$$

Then the condition (3.25) is obviously valid for $m=1$ in view of the positivity (3.12). For $m>1$, checking (3.25) is not straightforward since all the eigenfunctions are of changing sign. Anyway, we expect that (3.25) is always valid for constant data (3.27). It is curious that, for $m \geq 2$, this is an open problem.

3.5. On special solutions with fixed nodal sets. Using the separation (3.17), we look for special solutions

$$
u(x, t)=v(r, t) f_{k}(\sigma), \quad r=|x|,
$$

of the original singular equation (1.6) with $\Omega=B_{1}$. These solutions have fixed (time-independent) nodal sets that actually change the Hardy constant, since now 0 effectively belongs to an artificial boundary, on which $u=0$. Indeed, formally substituting (3.28) into (1.6) yields the following radial equation for $v(r, t)$ :

$$
v_{t}=(-1)^{m+1} \sum_{l=0}^{m} C_{m}^{l}\left(-\mu_{k}\right)^{m-l} \Delta_{r}^{l}\left(\frac{1}{r^{2(m-l)}} v\right)+\frac{c}{r^{2 m}} v .
$$

It follows that the last most singular at $y=0$ term is now

$$
\ldots+\frac{c-\left(\mu_{k}\right)^{m}}{r^{2 m}} v
$$

so that the problem becomes subcritical provided that

$$
c-\left(\mu_{k}\right)^{m} \leq c_{\mathrm{H}} \text {, i.e., for } c \leq c_{\mathrm{H}}+\left(\mu_{k}\right)^{m} \text {. }
$$

Thus, for any $c>c_{\mathrm{H}}$, there exist global solutions (3.28) of (1.6) with $k \gg 1$.

Note that this by no means undermines the phenomenon of the strong instability in the singular equation (1.6) in the supercritical range (1.9). Indeed, it is not clear which solutions (3.28), (3.31) do withstand the $\varepsilon$-regularization without blow-up as $\varepsilon \rightarrow 0$. We expect that, without the assumption $\Omega=B_{1}$ or without special symmetries of $\Omega$ supporting the fixed nodal set of special solutions under consideration, no 
solutions actually do. However, in the maximal generality, the proof is not a part of our business here. In Section 5, we present the proof of blow-up for some stationary data that are supposed to be the most resistive to regular $\varepsilon$-approximations, but actually are not.

\section{Constructing Data With a Doubly oscillatory $\pm \infty$ Limit}

In this section, for the reason of performing some rather involved calculus, we take the unit ball, $\Omega=B_{1}$, and restrict to radial solutions only, where $r=|x| \in$ $[0,1)$.

4.1. Singular stationary solution (SSS). Consider the radial stationary equation

$$
\mathbf{B}_{0} U \equiv-\left(-\Delta_{r}\right)^{m} U+\frac{c}{r^{2 m}} U=0 \quad \text { for } \quad r \in(0,1) .
$$

As usual for Euler-type ODEs, looking for solutions of (4.1),

$$
U(r)=r^{\gamma},
$$

we obtain the following characteristic equation for $\gamma \in \mathbb{C}$ :

$$
\begin{gathered}
G(\gamma) \equiv G_{*}(\gamma)+c=0, \quad \text { where } \\
G_{*}(\gamma)=(-1)^{m+1} \prod_{k=1}^{m}[\gamma-2(k-1)](\gamma+N-2 k) .
\end{gathered}
$$

Then (1.10) means that (see, e.g., [8])

$$
c=c_{\mathrm{H}}=-G_{*}\left(-\frac{N-2 m}{2}\right)>0,
$$

so the best Hardy constant $c_{\mathrm{H}}$ is such that the function (4.2) with the exponent

$$
\gamma=\gamma_{m}=-\frac{N-2 m}{2}
$$

is the exact weak solution of the homogeneous equation $\mathbf{B}_{0} \psi=0$ in $\mathbb{R}^{N}$. Thus, for $c=c_{\mathrm{H}}$, the characteristic equation has the double root (4.5). It generates two $L^{2}$-solutions,

$$
\bar{U}_{m}(r)=r^{-\frac{N-2 m}{2}} \ln r \quad \text { and } \quad \bar{U}_{m+1}(r)=r^{-\frac{N-2 m}{2}},
$$

which are ordered relative to the growth rate as $r \rightarrow 0$. Other $2 m-2$ characteristic roots of (4.3) are real or complex. Complex roots can occur for $m \geq 3$. One can see from the structure of the characteristic polynomial (4.3) that, for $N \gg 2 m$ and $m$ even, there exist precisely two more real roots $\hat{\gamma}_{m-1}>2(m-1)$ and $\gamma_{m-1}<2-N$. On the other hand, for $N \gg 2 m$ and $m$ odd, no more real roots exist; see [8].

It follows from (4.3) that in the supercritical range $c>c_{\mathrm{H}}$, the characteristic polynomial admits two complex roots,

$$
\gamma_{m \pm}=-\frac{N-2 m}{2} \pm \mathrm{i} d, \quad \text { where } \quad d=O\left(\sqrt{c-c_{\mathrm{H}}}\right)>0 .
$$

The corresponding solutions are oscillatory near the origin, e.g.,

$$
U_{m}(r)=r^{-\frac{N-2 m}{2}} \cos \left(d \ln r+a_{0}\right) \quad\left(a_{0} \in \mathbb{R}\right) .
$$

By a weak $S S S$ of (4.1) denoted by $U_{*}(r)$, we mean a solution that exhibits the oscillatory behaviour such as (4.8) as $r \rightarrow 0$. Note that

$$
U_{*} \in L^{p}(\Omega) \quad \text { for any } \quad p<\frac{2 N}{N-2 m} \text {. }
$$


4.2. $\varepsilon$-approximation diverges to $\pm \infty$. In the radial parabolic approximating problem for equation (1.12), we first take initial data $u_{0}(r)$ that are not continuous at $r=0$ and exhibit an oscillatory behaviour. It is convenient to assume the behaviour as in (4.8), i.e.,

$$
u_{0}(r)=r^{-\frac{N-2 m}{2}} \cos (d \ln r) \quad \text { for } r>0 \text { small } \quad\left(\text { or, simply, } u_{0}(r)=U_{*}(r)\right),
$$

though other types of oscillations will also suffice.

Let us explain the origin of such huge oscillatory properties of the approximation $u_{\varepsilon}(x, t)$. Using the same idea, we apply the estimates from Section 2 that are sufficient. The simpler case $m=1$, where $U_{0}(y)>0$ (see (3.12) $)$, was studied in [9], so here $m \geq 2$.

Thus, we begin with formal estimates of the first Fourier coefficient for the maximal positive eigenvalue as in (2.15) (here $r=|x|>0$ ):

$$
\lambda_{0}^{\varepsilon} \approx \frac{\Lambda_{0}}{\varepsilon^{2 m}} \quad \text { and } \quad \psi_{0}^{\varepsilon}(x) \sim \varepsilon^{-\frac{N}{2}} U_{0}\left(\frac{x}{\varepsilon}\right) \sim \varepsilon^{-\frac{N}{2}} \mathrm{e}^{-a_{m} \Lambda_{0}^{1 / 2 m} \frac{r}{\varepsilon}} \cos \left(b_{m} \frac{r}{\varepsilon}\right) \text { for } r \gg \varepsilon,
$$

where $\mu_{m}=\frac{1}{\varepsilon}\left(-a_{m}+\mathrm{i} b_{m}\right)$ is the root of $(-1)^{m+1} \mu^{2 m}=\frac{\Lambda_{0}}{\varepsilon^{2 m}}$ with the maximal $\operatorname{Re} \mu_{m}=-a_{m}<0$. This yields, in the first rough, but sufficient, approximation, the coefficient

$$
c_{0}^{\varepsilon}=\left\langle u_{0}, \psi_{0}^{\varepsilon}\right\rangle \sim \varepsilon^{-\frac{N}{2}} \int_{0}^{\frac{1}{\varepsilon}} r^{N-1} u_{0}(r) U_{0}\left(\frac{r}{\varepsilon}\right) \mathrm{d} r=\varepsilon^{\frac{N}{2}} \int_{0}^{\frac{1}{\varepsilon^{2}}} z^{N-1} u_{0}(\varepsilon z) U_{0}(z) \mathrm{d} z .
$$

Substituting the next data (4.10) yields the following estimate of the first Fourier coefficient:

$$
c_{0}^{\varepsilon} \sim \varepsilon^{m} \int_{0}^{+\infty} z^{\frac{N+2 m-2}{2}} U_{0}(z) \cos (d \ln z-D) \mathrm{d} z \equiv \varepsilon^{m} I_{d}(D),
$$

where $D=-d \ln \varepsilon \rightarrow+\infty$ as $\varepsilon \rightarrow 0$ is a shifting argument in $\cos (\cdot)$, which changes the sign of the function in the integral. Recall that $U_{0}(z)$ is exponentially small by (4.11) as $z \rightarrow \infty$, so the integral in (4.13) fast converges. Here, we assume that both integrals

$$
\int_{0}^{+\infty} z^{\frac{N+2 m-2}{2}} U_{0}(z) \cos (d \ln z) \mathrm{d} z \quad \text { and } \quad \int_{0}^{+\infty} z^{\frac{N+2 m-2}{2}} U_{0}(z) \sin (d \ln z) \mathrm{d} z
$$

do not vanish simultaneously (otherwise, we change $d$ and/or the exponent $-\frac{N-2 m}{2}$ or any other parameters to get necessary nonzero values). Then, in view of the analytic dependence on parameters in (4.13), for any fixed $d>0$ there exist sequences $\left\{\varepsilon_{k}^{ \pm}\right\} \rightarrow 0$ such that $c_{0}^{\varepsilon_{k}^{+}} \geq\left(\varepsilon_{k}^{+}\right)^{m} \delta_{0}$ and $c_{0}^{\varepsilon_{k}^{-}} \leq-\left(\varepsilon_{k}^{-}\right)^{m} \delta_{0}$ for any $k=0,1,2, \ldots$ with some constant $\delta_{0}>0$. Therefore, by (4.11), for $\varepsilon=\varepsilon_{k}^{ \pm} \rightarrow 0$,

$$
u_{\varepsilon}\left(x, t_{0}\right) \sim c_{0}^{\varepsilon} \varepsilon^{m-\frac{N}{2}} I_{d}(d \ln \varepsilon) \mathrm{e}^{-a_{m} \Lambda_{0}^{1 / 2 m} \frac{r}{\varepsilon}} \cos \left(b_{m} \frac{r}{\varepsilon}\right) \mathrm{e}^{\Lambda_{0} \frac{t_{0}}{\varepsilon^{2} m}} \quad(r=|x|),
$$

where we may assume that $\cos (\cdot)$ does not violate the above sign restrictions on $c_{0}^{\varepsilon}$. Similarly, in view of the oscillatory functions $\cos \left(b_{m} \frac{r}{\varepsilon}\right)$ in (4.14), there exist sequences $\left\{\hat{\varepsilon}_{k}^{ \pm}\right\} \rightarrow 0$ such that $\psi_{0}^{\varepsilon}(x)$ stands positive and negative, respectively.

Thus, we observe a doubly oscillatory singular limit to $\pm \infty$ as $\varepsilon \rightarrow 0$ for the regularizing solution (4.14). Imposing necessary elementary assumptions on these four sequences $\left\{\varepsilon_{k}^{ \pm}\right\}$and $\left\{\hat{\varepsilon}_{k}^{ \pm}\right\}$, one can always choose some "intermediate" sequences $\left\{\bar{\varepsilon}_{k}^{ \pm}\right\} \rightarrow 0$ along which the limits $\pm \infty$ are guaranteed. Thus, by (4.14), we observe that, for any $r=|x|>0$, in the sense of the first Fourier coefficient,

$$
\limsup _{\varepsilon \rightarrow 0} u_{\varepsilon}(x, t)=+\infty \text { and } \liminf _{\varepsilon \rightarrow 0} u_{\varepsilon}(x, t)=-\infty .
$$


In particular, we also easily fix the following weaker $\pm \infty$-divergence:

$$
\limsup \sup _{\varepsilon \rightarrow 0} \sup _{x} u_{\varepsilon}(x, t)=+\infty \quad \text { and } \quad \liminf _{\varepsilon \rightarrow 0} \inf _{x} u_{\varepsilon}(x, t)=-\infty .
$$

It is interesting that, by oscillatory behaviour of the Fourier coefficient $c_{0}^{\varepsilon}$ in (4.13), the nonuniform divergence (4.15) holds even at the origin $x=0$.

On the other hand, if for given data $u_{0}$ the coefficient $c_{0}^{\varepsilon}$ is not oscillatory at all, e.g.,

$$
c_{0}^{\varepsilon} \geq c_{\varepsilon}>0 \text { for all small } \varepsilon>0,
$$

the oscillatory divergence (4.16) or (4.15) for any $x \neq 0$ holds due to the signchanging behaviour of $U_{0}(y)$ for $y \gg 1$ given in (4.11).

\section{An ANALYTIC Stationary PROFILE BLOWs UP AS $\varepsilon \rightarrow 0$}

Let us present a different simple example showing another unstability feature of the $\varepsilon$-approximation of (1.6), which we now consider in $\mathbb{R}^{N} \times(0, T)$; for convenience, we pose the Cauchy problem. We choose $c>c_{\mathrm{H}}$ such that the characteristic equation (4.3) has the root $\gamma=2 m$, so that (4.1) has the analytic stationary solution

$$
U_{0}(x)=|x|^{2 m} \text { in } \mathbb{R}^{N} .
$$

Consider the regularized equation (1.12) with the stationary data (5.1). Choosing, for convenience, the new variable

$$
v=u_{t}
$$

and differentiating (1.12) in $t$, we obtain for $v$ the same equation (1.12) with data

$$
\begin{aligned}
v_{0 \varepsilon}(x) & =\mathbf{B}_{\varepsilon}|x|^{2 m} \equiv-(-\Delta)^{m}|x|^{2 m}+\frac{c}{\varepsilon^{2 m}+|x|^{2 m}}|x|^{2 m} \\
& \equiv c\left(\frac{1}{\varepsilon^{2 m}+|x|^{2 m}}-\frac{1}{|x|^{2 m}}\right)=-\frac{c \varepsilon^{2 m}}{\varepsilon^{2 m}+|x|^{2 m}} .
\end{aligned}
$$

After scaling (3.1), we arrive at equation (3.5) with $\varepsilon$-independent initial data

$$
v_{0}(y)=-\frac{c}{1+|y|^{2}}<0 \quad \text { in } \quad \mathbb{R}^{N} .
$$

Therefore, (3.25) guarantees blow-up as $\varepsilon \rightarrow 0$. As usual, $m=1$ is easy by (3.12).

Thus, in general, even classical stationary solutions of the singular equation (1.6) do not stand $\varepsilon$-regularization, to say nothing about other more arbitrary (nonsteady) data.

\section{Parabolic extensions: Time-DePendent and nOnlinear potentials}

6.1. Time-dependent potentials: Towards nonself-adjoint spectral theory and a new Hardy constant. New spectral phenomena appear when the potential in (2.1) depends on the time-variable in an $\varepsilon$-scaling manner, e.g., as in equation with the following singular critical potential (cf. (1.6)):

$$
u_{t}=-(-\Delta)^{m} u+\frac{c}{t+|x|^{2 m}} u \text {. }
$$

Then the natural regularized equation reads

$$
u_{\varepsilon}: \quad u_{t}=-(-\Delta)^{m} u+\frac{c}{\varepsilon^{2 m}+t+|x|^{2 m}} u .
$$

As usual, we treat a general regularized equation with such potentials,

$$
u_{\varepsilon}: \quad u_{t}=\mathbf{B}_{\varepsilon} u=-(-\Delta)^{m} u+\frac{1}{\varepsilon^{2 m}} q\left(\frac{x}{\varepsilon}, \frac{t}{\varepsilon^{2 m}}\right) u \quad \text { in } \quad \mathbb{R}^{N} \times \mathbb{R}_{+} .
$$


For convenience and as a different example, we now ignore boundary conditions and consider the Cauchy problem with data $u_{0 \varepsilon} \in L^{2}\left(\mathbb{R}^{N}\right) \cap L^{\infty}\left(\mathbb{R}^{N}\right)$. Scaling (3.1) yields for

$$
u_{\varepsilon}(x, t)=v_{\varepsilon}\left(\frac{x}{\varepsilon}, \frac{t}{\varepsilon^{2 m}}\right)
$$

the following rescaled equation:

$$
v_{\varepsilon}: \quad v_{\tau}=-(-\Delta)^{m} v+q(y, \tau) v, \quad v(y, 0)=v_{0 \varepsilon}(y)=u_{0 \varepsilon}(\varepsilon y)
$$

Since by (6.4) the behaviour of $\left\{u_{\varepsilon}\right\}$ as $\varepsilon \rightarrow 0$ is equivalent to the behaviour of $\left\{v_{\varepsilon}(y, \tau)\right\}$ as $\tau \rightarrow+\infty$, we perform extra scaling and introduce the new standard similarity variable from the heat kernel of the operator $D_{t}+(-\Delta)^{m}$ :

$$
z=\frac{y}{(1+\tau)^{1 / 2 m}}, \quad s=\ln (1+\tau) \rightarrow+\infty .
$$

Then $w_{\varepsilon}(z, s)=v_{\varepsilon}(y, \tau)$ solves the equation

$$
w_{\varepsilon}: \quad w_{s}=-(-\Delta)^{m} w+\frac{1}{2 m} z \cdot \nabla w+\mathrm{e}^{s} q\left(z \mathrm{e}^{\frac{s}{2 m}}, \mathrm{e}^{s}-1\right) w
$$

In order to approach a fixed regularized potential (as in (3.5)), we assume that

$$
\mathrm{e}^{s} q\left(z \mathrm{e}^{\frac{s}{2 m}}, \mathrm{e}^{s}-1\right) \rightarrow \frac{c}{1+|z|^{2 m}} \quad \text { as } \quad s \rightarrow+\infty \text { uniformly }
$$

and sufficiently fast. For instance, (6.8) holds for the potential in (6.2) since

$$
\frac{1}{\varepsilon^{2 m}} q\left(\frac{x}{\varepsilon}, \frac{t}{\varepsilon^{2 m}}\right)=\frac{c}{\varepsilon^{2 m}+t+|x|^{2 m}} \quad \Longrightarrow \quad q(y, \tau)=\frac{c}{1+\tau+|y|^{2 m}} .
$$

Thus, passing to the limit in (6.7) with the assumption (6.8), we obtain the equation

$$
w_{s}=-(-\Delta)^{m} w+\frac{1}{2 m} z \cdot \nabla w+\frac{c}{1+|z|^{2 m}} w
$$

Here the limit operator

$$
\mathbf{B}_{1} \equiv-(-\Delta)^{m}+\frac{1}{2 m} z \cdot \nabla+\frac{c}{1+|z|^{2 m}} I
$$

admits Friedrichs' self-adjoint extension for the second-order case $m=1$ only, where it possesses a symmetric representation of the form

$$
\mathbf{B}_{1} \equiv \frac{1}{\rho} \nabla \cdot(\rho \nabla)+\frac{c}{1+|z|^{2 m}} I \quad \text { in } \quad L_{\rho}^{2}\left(\mathbb{R}^{N}\right), \text { with } \quad \rho(z)=\mathrm{e}^{\frac{|z|^{2}}{4}} .
$$

For $m>1$, 6.11) is not self-adjoint and is not symmetric in any weighted $L^{2}$-spaces.

Consider the linear operator with the same principal differential part,

$$
\mathbf{B}=-(-\Delta)^{m}+\frac{1}{2 m} z \cdot \nabla+\frac{N}{2 m} I
$$

It is known [6] that, for any $m>1$, operator (6.13) is naturally defined in the weighted space $L_{\rho}^{2}\left(\mathbb{R}^{N}\right)$, with

$$
\rho(y)=\mathrm{e}^{a|y|^{\alpha}}, \quad \text { with } \quad \alpha=\frac{2 m}{2 m-1},
$$

where $a>0$ is a sufficiently small constant. The domain of $\mathbf{B}$ is the corresponding Sobolev space $H_{\rho}^{2 m}\left(\mathbb{R}^{N}\right)$, so that $\mathbf{B}: H_{\rho}^{2 m}\left(\mathbb{R}^{N}\right) \rightarrow L_{\rho}^{2 m}\left(\mathbb{R}^{N}\right)$ is bounded with discrete spectrum

$$
\sigma(\mathbf{B})=\left\{-\frac{l}{2 m}, l=0,1,2, \ldots\right\} .
$$

In particular, both $\mathbf{B}$ and $\mathbf{B}^{*}$ then have discrete spectra, compact resolvents, and complete sets of eigenfunctions. Some of these results can be extended to the operator (6.11), for which spectral theory deserves further development.

We now concentrate on particular spectral properties related to the nonexistence. 
CONSIDER FIRST THE SELF-ADJOINT CASE $m=1$. Then, by classic theory 2, 20], there exists a monotone branch $\Lambda_{0}=\Lambda_{0}(c)$ of the first simple real eigenvalue of $\mathbf{B}_{1}$ (here $m=1$ ):

$$
\Lambda_{0}(0)=-\frac{N}{2 m} \quad \text { and } \quad \Lambda_{0}(c) \rightarrow+\infty \text { as } c \rightarrow+\infty .
$$

We then define the new "Hardy constant" for the $c$-family of operators (6.11) as follows:

$$
c_{\mathrm{H}}: \quad \Lambda_{0}(c)=0 \in \sigma\left(\mathbf{B}_{1}\right) .
$$

Then, for any $c>c_{\mathrm{H}}$, there exists $\Lambda_{0}(c)>0$ that leads to a generic blow-up of approximations $\left\{u_{\varepsilon}\right\}$, provided that $v_{0}$ is not orthogonal to $U_{0}$. Analogously, further increasing $c>c_{\mathrm{H}}$ leads to the appearance of more branches of positive eigenvalues $\Lambda_{1}(c), \ldots, \Lambda_{K}(c)$, so that we can have the positive lineal $\mathcal{L}_{+}=\operatorname{Span}\left\{U_{0}, \ldots, U_{K}\right\}$ of arbitrarily large, but finite dimension $K=K(c)$ for $c \gg 1$.

Thus, taking into account the first unstable mode only, we have from scalings (6.4) and (6.6) that, under the "nonorthogonality assumption" to $U_{0} \in \mathcal{L}_{+}$, the following divergence rate is achieved: for an arbitrarily small fixed $t>0$ (here again $m=1$ ),

$$
u_{\varepsilon}(x, t) \sim\left(\frac{t}{\varepsilon^{2 m}}\right)^{\Lambda_{0}(c)} U_{0}\left(\frac{x}{t^{1 / 2 m}}\right) \text { as } \varepsilon \rightarrow 0 .
$$

Similar to Theorem 2.1. the behaviour (6.18) is easy to characterize in terms of $L^{p}\left(\mathbb{R}^{N}\right)$-divergence for any $p \geq 1$, as well as in other metrics.

On the contrary, for $c \leq c_{\mathrm{H}}$ the family $\left\{u_{\varepsilon}=v_{\varepsilon}=w_{\varepsilon}\right\}$ is uniformly bounded, meaning the existence of a bounded solution (of (6.1), say).

Consider $m \geq 2$, where we can guarantee less. Since in (6.11) the last term $\frac{c}{1+|z|^{2 m}} I$ serves as a compact perturbation of $\mathbf{B}$ in the equivalent integral representation, we still have a discrete spectrum and a countable family of continuous eigenvalue branches for all $c \geq 0$. For $c=0$, there holds

$$
\mathbf{B}_{1}=\mathbf{B}-\frac{N}{2 m} I
$$

so by perturbation methods [20, we find the local branch as in (6.16) of the first real simple eigenvalue $\Lambda_{0}(c)$ of $\mathbf{B}_{1}$ for all sufficiently small $c>0$. Further extension of the branch in this nonself-adjoint case is not guaranteed to be strictly monotone, and the eigenvalue does not necessarily always remain real. Then we are assumed to deal with the first eigenvalue having the maximal real part (see (6.17)),

$$
\operatorname{Re} \Lambda_{0}(c)>0 \text { for } c>c_{\mathrm{H}} \text {. }
$$

This definition is based on a natural property that $\operatorname{Re} \Lambda_{0}(c)$ grows unboundedly as $c \rightarrow+\infty$, though passage to the limit $c \rightarrow+\infty$ in the family (6.11) is quite tricky and not studied here. Then under the assumption (6.19) (or 6.17) in the real case), we still obtain the divergence result of the type (6.18) for any nonorthogonal initial data in the sense that $\left\langle v_{0}, U_{0}^{*}\right\rangle \neq 0$, where $U_{0}^{*}$ is the corresponding adjoint eigenfunction of $\mathbf{B}_{1}^{*}$.

Thus, for all $m \geq 1$, according to our previous results, the divergence of the family $\left\{u_{\varepsilon}\right\}$ as $\varepsilon \rightarrow 0$ will be guaranteed under the hypothesis of the type (3.25), where $\mathcal{L}_{+}$is the positive lineal of the limit operator (6.11). Indeed, deeper spectral analysis of the nonself-adjoint operators (6.11) are quite in demand here. 
6.2. On nonlinear parabolic equations. As a next natural extension, we consider the following semilinear parabolic equation with the same singular potential as in (1.6):

$$
u_{t}=-(-\Delta)^{m} u+\frac{1}{|x|^{2 m}}|u|^{p}, \quad \text { where } \quad p>1 .
$$

Here, the nonlinearity is taken in the form $|u|^{p}$ for convenience of further simple calculus in the case $m \geq 2$, when the Maximum Principle fails.

The condition $p>1$ involves the extra high instability of the evolution driven by the reaction-diffusion PDE (6.20), since now it describes blow-up of solutions in finite time. For $m=1$ and $u_{0} \geq 0$, the nonexistence results for (6.20) were obtained in Brezis-Cabré [3]; for the full history and extensions see [16, 22], [7, p. 333], and [25. pp. 108, 267]. For solutions of changing sign, the nonexistence results for (6.20) were absent even for $m=1$.

Let us see how the nonlinearity $|u|^{p}$ affects the regularized solutions satisfying, as usual,

$$
u_{\varepsilon}: \quad u_{t}=-(-\Delta)^{m} u+\frac{1}{\varepsilon^{2 m}+|x|^{2 m}}|u|^{p} .
$$

Then scaling (3.1) yields

$$
v_{\varepsilon}: \quad v_{t}=-(-\Delta)^{m} v+\frac{1}{1+|y|^{2 m}}|v|^{p} \quad \text { in } \quad \mathbb{R}^{N} \times \mathbb{R}_{+},
$$

and for simplicity we assume that data are constant as in (3.27).

Case $m=1$. Then the proof of blow-up of (6.22) is straightforward, since by (3.27),

$$
v_{t}>0 \text { in } \mathbb{R}^{N} \times \mathbb{R}_{+}
$$

via the Maximum Principle. Therefore, the solution $v(y, \tau)$ is strictly monotone increasing in $t$ and is not bounded above (since otherwise, as for gradient systems, $v(\tau)$ would stabilize to a bounded steady solution, which is nonexistent); see details in [7, p. 319]. Denoting by $T_{*}$ the blow-up time for (6.22), we take into account the fact that due to (6.23) blow-up is complete; i.e., in the natural sense of the proper minimal extension,

$$
v(y, \tau) \equiv+\infty \quad \text { for } \quad \tau>T_{*} .
$$

We then conclude that, for all small $\varepsilon>0$,

$$
u_{\varepsilon}(x, t) \text { blows up completely in time } T_{\varepsilon}<2 \varepsilon^{2 m} T_{*} \text {. }
$$

This ends the proof of divergence as $\varepsilon \rightarrow 0$ of $\left\{u_{\varepsilon}\right\}$ under the assumption (3.26) for $m=1$.

Case $m \geq 2$. A similar scheme applies with some changes since (6.23) is no longer true. Finite time blow-up in (6.22), (3.27) is then proved by the eigenfunction method that shows blow-up of the first Fourier coefficient

$$
E(\tau)=\left\langle v(y, \tau), \psi_{1}\left(\frac{y}{R}\right)\right\rangle \quad(R \gg 1),
$$

where $\psi_{1}(z)$ is a specially constructed nonnegative cut-off function satisfying the following elliptic inequality: there exists a constant $\lambda_{1}>0$ such that

$$
\left|\Delta^{m} \psi_{1}\right| \leq \lambda_{1} \psi_{1} \quad \text { in } \mathbb{R}^{N} .
$$

See a convenient adaptation of Kaplan's eigenfunction method (1963) to higherorder parabolic operators in [10, §5]. It is crucial that the resulting ordinary differential inequality for $E(\tau)$ implies not only the fact of finite-time blow-up at some $T_{*}$, but also that it becomes complete (possibly, at some moment $T_{\mathrm{c}}>T_{*}$ ). An 
alternative proof of blow-up in (6.22) by the nonlinear capacity method can be performed as in [25, §29]. This leads to the nonexistence conclusion (6.24). Here, similar to (2.15), we control the complete blow-up of the "first Fourier coefficient" relative to a solution $\psi_{1}$ of the elliptic inequality (6.25).

The above scheme on nonexistence applies to the truly quasilinear parabolic model

$$
u_{\varepsilon}: \quad u_{t}=-(-\Delta)^{m}\left(|u|^{n} u\right)+\frac{1}{\varepsilon^{2 m}+|x|^{2 m}}|u|^{n+1}, \quad \text { where } \quad n>0 .
$$

\section{ON NONEXISTENCE FOR OTHER LINEAR AND SEMILINEAR PDES}

7.1. Schrödinger-type equations. A typical linear example is

$$
u_{\varepsilon}: \quad \mathrm{i} u_{t}=\mathbf{B}_{\varepsilon} u=-(-\Delta)^{m} u+\frac{c}{\varepsilon^{2 m}+|x|^{2 m}} u .
$$

The principle difference is that all $u_{\varepsilon}$ satisfy the conservation law

$$
\left\|u_{\varepsilon}(t)\right\|_{L^{2}}=\left\|u_{0 \varepsilon}\right\|_{L^{2}} \quad \text { for all } t>0 .
$$

Nevertheless, the nonexistence conclusion for $c>c_{\mathrm{H}}$ is derived in a similar manner, though, in view of (7.2), the key feature of divergence is that the family $\left\{u_{\varepsilon}(x, t)\right\}$ gets extremely oscillatory as $\varepsilon \rightarrow 0$ in the variable $t>0$. Indeed, now, loosely speaking, for positive eigenvalues $\Lambda_{j}$ of $\mathbf{B}_{1}$,

$$
\lambda_{j}^{\varepsilon} \sim-\mathrm{i} \frac{\Lambda_{j}}{\varepsilon^{2 m}} \text { for small } \varepsilon>0 .
$$

Finally, instead of (2.15), in view of the oscillatory behaviour with purely imaginary eigenvalues (7.3), assuming the strict inequality $\Lambda_{0}>\Lambda_{1}$ (cf. (3.10), we suggest the following characterization of the actual divergence of solution (of course this is just a formal illustration that is not that informative, which any isolated mode admits):

$$
\mathrm{e}^{\mathrm{i} \lambda_{0}^{\varepsilon} t} u_{\varepsilon}(x, t)-c_{0}^{\varepsilon} \psi_{0}^{\varepsilon}\left(\frac{x}{\varepsilon}\right) \rightarrow 0 \quad \text { as } \varepsilon \rightarrow 0 \quad \text { weakly in } L_{\text {loc }}^{2}(\Omega \times(0, \delta)) .
$$

Thus, in terms of $u_{\varepsilon}$, we get rather weak blow-up. However, the time-derivative $\left(u_{\varepsilon}\right)_{t}$ then truly blows up as $\varepsilon \rightarrow 0$ according to

$$
\left(u_{\varepsilon}\right)_{t}(x, t) \sim \frac{1}{\varepsilon^{2 m}} \mathrm{e}^{-\mathrm{i} \lambda_{0}^{\varepsilon} t} c_{0}^{\varepsilon} \psi_{0}^{\varepsilon}\left(\frac{x}{\varepsilon}\right) .
$$

The real parts of the solutions, $u_{\varepsilon}=U_{\varepsilon}+\mathrm{i} V_{\varepsilon}$, satisfy a $4 m$ th-order hyperbolic equation,

$$
U_{\varepsilon}: \quad U_{t t}=-\mathbf{B}_{\varepsilon}^{2} U,
$$

to which a general scheme of the analysis, with some changes, can be also applied.

As usual, a similar "nonexistence" analysis of the corresponding nonlinear Schrödinger equation (NLS)

$$
u_{\varepsilon}: \quad \text { i } u_{t}=\mathbf{B}_{\varepsilon} u \equiv-(-\Delta)^{m} u+\frac{1}{\varepsilon^{2 m}+|x|^{2 m}}|u|^{p-1} u \quad(p>1)
$$

demands extra results on blow-up in the corresponding rescaled equation

$$
v_{\varepsilon}: \quad \mathrm{i} v_{\tau}=\mathbf{B}_{1} v \equiv-(-\Delta)^{m} v+\frac{1}{1+|y|^{2 m}}|v|^{p-1} v .
$$

For $m=1$, blow-up for such NLSEs in some parameter ranges of $p$ and $N$ is a classic matter of the theory and application to nonlinear optics, plasma physics, and others; see references to key papers and monographs on this subject in MerleRaphael 24] and in survey [12]. The higher-order case $m \geq 2$ is less developed, though some techniques can be applied. Notice that we are interested in a quite 
particular setting with constant data as in (3.27), for which the proof of blow-up seems not very difficult.

Thus, we will get typical "divergence" of the family $\left\{u_{\varepsilon}\right\}$ as $\varepsilon \rightarrow 0$, provided that the suitable rescaled solution of (7.6) blows up in finite time. Note that finite $L^{2}$-energy blow-up solutions of NLSEs admit a natural extension beyond blow-up time, i.e., for $t>T$ in view of the conservation (17.2) (possibly, this is not the case for data (3.27) of infinite energy). Then the divergence of $\left\{u_{\varepsilon}\right\}$ as $\varepsilon \rightarrow 0$ might not be as lethal as in other examples, so again a further study of such singularity phenomena is necessary.

7.2. Hyperbolic equations. A key regularized linear model is constructed analogously,

$$
u_{\varepsilon}: \quad u_{t t}=\mathbf{B}_{\varepsilon} u \equiv-(-\Delta)^{m} u+\frac{c}{\varepsilon^{2 m}+|x|^{2 m}} u,
$$

with initial data $u_{0}, u_{1}$. Taking $t=\varepsilon^{m} \tau$ in (3.1) yields the rescaled equation

$$
v_{\varepsilon}: \quad v_{\tau \tau}=\mathbf{B}_{1} v \equiv-(-\Delta)^{m} v+\frac{c}{1+|y|^{2 m}} v .
$$

The separation of variables generates the eigenvalue problem (2.9), where $\lambda \mapsto \lambda^{2}$. Hence, the positive lineal of $\mathbf{B}_{1}$ continues to play the key role for the nonexistence as $\varepsilon \rightarrow 0$, so under similar nonorthogonality assumptions we arrive at the divergence result such as (2.15), with $\sqrt{\lambda_{0}^{\varepsilon}}$ in the first multiplier.

The corresponding nonlinear hyperbolic equation,

$$
u_{\varepsilon}: \quad u_{t t}=\mathbf{B}_{\varepsilon} u \equiv-(-\Delta)^{m} u+\frac{1}{\varepsilon^{2 m}+|x|^{2 m}}|u|^{p},
$$

leads to the rescaled PDE

$$
v_{\varepsilon}: \quad v_{\tau \tau}=\mathbf{B}_{1} v \equiv-(-\Delta)^{m} v+\frac{1}{1+|y|^{2 m}}|v|^{p} .
$$

For data (3.27), the necessary blow-up results for (7.10) can be obtained as in 25, Ch. 5-7], so that the nonexistence-divergence conclusions as $\varepsilon \rightarrow 0$ persist in a similar way.

\section{REFERENCES}

[1] P. Baras and J.A. Goldstein, The heat equation with a singular potential, Trans. Amer. Math. Soc., 284 (1984), 121-139. MR742415 (85f:35099)

[2] M.S. Birman and M.Z. Solomjak, Spectral Theory of Self-Adjoint Operators in Hilbert Space, D. Reidel, Dordrecht/Tokyo, 1987.

[3] H. Brezis and X. Cabré, Some simple non-linear PDEs without solutions, Boll. U.M.I., serie VIII, I-B (1998), 223-262. MR1638143 (99j:35001)

[4] H. Brezis and M. Marcus, Hardy's inequalities revisited, Ann. Scuola Norm. Sup. Pisa Cl. Sci. (4), 25 (1998), 217-237. MR.1655516 (99m:46075)

[5] H. Brezis and J.L. Vazquez, Blow-up solutions of some nonlinear elliptic equations, Revista Mat. Complutense, 10 (1997), 443-469. MR.1605678 (99a:35081)

[6] Yu.V. Egorov, V.A. Galaktionov, V.A. Kondratiev, and S.I. Pohozaev, Global solutions of higher-order semilinear parabolic equations in the supercritical range, Adv. Differ. Equat., 9 (2004), 1009-1038. MR2098064 (2005g:35132)

[7] V.A. Galaktionov, Geometric Sturmian Theory of Nonlinear Parabolic Equations and Applications, Chapman and Hall/CRC, Boca Raton, Florida, 2004. MR2059317 (2005h:35002)

[8] V.A. Galaktionov, On extensions of higher-order Hardy's inequalities, Differ. Integr. Equat., 19 (2006), 327-344. MR2215561 (2007b:26039)

[9] V.A. Galaktionov, On nonexistence result of Baras-Goldstein type for singular linear and nonlinear parabolic equations without positivity assumptions, Proc. Steklov Math. Inst., 260 (2008), 130-150. 
[10] V.A. Galaktionov and S.I. Pohozaev, Existence and blow-up for higher-order semilinear parabolic equations: majorizing order-preserving operators, Indiana Univ. Math. J., 51 (2002), 1321-1338. MR 1948452 (2003k:35104)

[11] V.A. Galaktionov and J.L. Vazquez, Continuation of blow-up solutions of nonlinear heat equations in several space dimensions, Comm. Pure Appl. Math., 50 (1997), 1-68. MR.1423231 (97h:35085)

[12] V.A. Galaktionov and J.L. Vazquez, The problem of blow-up in nonlinear parabolic equations, Discr. Cont. Dyn. Syst., 8 (2002), 399-433. MR1897690 (2003c:35067)

[13] F. Gazola, H.-C. Grunau, and E. Mitidieri, Hardy inequalities with optimal constants and remainder terms, Trans. Amer. Math. Soc., 356 (2004), 2149-2168. MR2048513 (2005c:26031)

[14] N. Ghoussoub and X.S. Kang, Hardy-Sobolev critical elliptic equations with boundary singularities, Ann. Inst. H. Poincaré-AN, 21 (2004), 767-793. MR2097030 (2005i:35086)

[15] N. Ghoussoub and F. Robert, The effect of curvature on the best constant in the HardySobolev inequalities, Geom. Funct. Anal., 16 (2006), 1201-1245. MR2276538 (2007k:35085)

[16] G.R. Goldstein, J.A. Goldstein, and I. Kombe, Nonlinear parabolic equations with singular coefficients and critical exponents, Appl. Anal., 84 (2005), 571-583. MR2151669(2006a:35150)

[17] J.A. Goldstein and Q.S. Zhang, On a degenerate heat equation with a singular potential, J. Funct. Anal., 186 (2001), 342-359. MR.1864826 (2002k:35179)

[18] J.A. Goldstein and Q.S. Zhang, Linear parabolic equations with strong singular potentials, Trans. Amer. Math. Soc., 355 (2002), 197-211. MR.1928085 (2003h:35096)

[19] M. Hoffman-Ostenhof, T. Hoffman-Ostenhof, and A. Laptev, A geometric version of Hardy's inequality, J. Funct. Anal., 189 (2002), 539-548. MR.1892180 (2003c:26022)

[20] T. Kato, Perturbation Theory for Linear Operators, Springer-Verlag, Berlin/New York, 1976. MR0407617 (53:11389)

[21] I. Kombe, The linear heat equation with highly oscillating potential, Proc. Amer. Math. Soc., 132 (2004), 2683-2691. MR2054795 (2005c:35129)

[22] I. Kombe, Nonlinear degenerate parabolic equations for Baouendi-Grushin operators, Math. Nachr., 279 (2006), 756-773. MR2226410 (2007f:35141)

[23] V. Maz'ja, Sobolev Spaces, Springer-Verlag, Berlin/Tokyo, 1985. MR817985 (87g:46056)

[24] F. Merle and P. Raphael, On a sharp lower bound on the blow-up rate for the $L^{2}$ critical nonlinear Schrödinger equation, J. Amer. Math. Soc., 19 (2005), 37-90. MR2169042 (2006j:35223)

[25] E. Mitidieri and S.I. Pohozaev, Apriori Estimates and Blow-up of Solutions to Nonlinear Partial Differential Equations and Inequalities, Proc. Steklov Inst. Math., Vol. 234, Intern. Acad. Publ. Comp. Nauka/Interperiodica, Moscow, 2001.

[26] J.L Vazquez and E. Zuazua, The Hardy inequality and the asymptotic behaviour of the heat equation with an inverse-square potential, J. Funct. Anal., 173 (2000), 103-153. MR.1760280 (2001j:35122)

[27] M.I. Vishik and A.A. Lyusternik, Regular degeneration and boundary layer for linear differential equations with small parameter, Uspehi Mat. Nauk (N.S.), 12 (1957), No. 5 (77), 3-122. MR.0096041 (20:2539)

[28] V.S. Vladimirov, Equations of Mathematical Physics, Marcel Dekker, Inc., New York, 1971. MR0268497 (42:3394)

[29] D. Yafaev, Sharp constants in the Hardy-Rellich inequalities, J. Funct. Anal., 168 (1999), 121-144. MR.1717839 (2001e:26027)

Department of Mathematical Sciences, University of Bath, Bath BA2 7Ay, United KINGDOM

E-mail address: vag@maths.bath.ac.uk

Department of Mathematical Sciences, University of Bath, Bath BA2 7Ay, United KINGDOM

E-mail address: ivk20@maths.bath.ac.uk 